\title{
Summary of the 1st AIAA Geometry and Mesh Generation Workshop (GMGW-1) and Future Plans
}

\author{
John R. Chawner ${ }^{1}$ \\ Pointwise, Fort Worth, Texas, 76104 \\ Todd Michal ${ }^{2}$ \\ Boeing Research and Technology, St Louis, MO, 63385 \\ Jeffrey P. Slotnick ${ }^{3}$ \\ The Boeing Company, Seattle, WA, 98124 \\ and \\ Christopher L. Rumsey ${ }^{4}$ \\ NASA Langley Research Center, Hampton, Virginia 23681, USA
}

\begin{abstract}
The 1st AIAA Geometry and Mesh Generation Workshop (GMGW-1) was held in conjunction with the AIAA Aviation Forum and Exposition 2017 and in collaboration with the 3rd AIAA Computational Fluid Dynamics (CFD) High Lift Prediction Workshop (HiLiftPW-3). As the first AIAA workshop on these topics, GMGW-1's broad objectives were to assess the current state-of-the art in geometry preprocessing and mesh generation technology as well as software as applied to aircraft and spacecraft systems. The workshop was intended to identify and develop understanding of areas of needed improvement in terms of performance, accuracy, and applicability. It was also to provide a foundation for documenting best practices for geometry preprocessing and mesh generation. The genesis of GMGW-1 is found in the indictments levied against geometry preprocessing and mesh generation - not undeservedly - by the NASA CFD Vision 2030 Study. In order to create a reference against which future progress in geometry preprocessing and mesh generation can be measured, the organizers of GMGW-1, with the assistance of the organizers of HiLiftPW3, focused GMGW-1 on generation of meshes of the NASA High Lift Common Research Model (HL-CRM). Some of the generated meshes were provided for use by the participants in HiLiftPW-3. All meshes and the processes by which they were generated were analyzed by GMGW-1 as a first assessment of state of the art practices. The results of GMGW-1 added quantitative detail to known problem areas including geometry modeling, data interoperability, and amount of human intervention. They do provide a clear path toward a vision of geometry preprocessing and mesh generation in the year 2030. The next milepost along this path will be a second workshop.
\end{abstract}

\section{Introduction}

A. CFD Vision 2030 and Mesh Generation

The CFD Vision 2030 Study: “A Path to Revolutionary Computational Aerosciences” was published in 2014 (Reference 1, hereinafter referred to as the Study). Its authors described the current state-of-the-art in CFD technology, developed a comprehensive roadmap including the expected maturation of key technology elements,

\footnotetext{
${ }^{1}$ President, 213 S. Jennings Ave., AIAA Associate Fellow.

2 Technical Fellow, P.O. Box 516 M/C S306-4030, AIAA Senior Member.

${ }^{3}$ Technical Fellow, Flight Sciences, P.O. Box 3707 M/C 0R-120, AIAA Associate Fellow.

${ }^{4}$ Senior Research Scientist, Computational AeroSciences Branch, Mail Stop 128, AIAA Fellow.
} 
and identified significant technical impediments that must be overcome if CFD is to be effectively utilized on the challenging applications the aerospace community will face by the year 2030. The vision of CFD in 2030 emphasizes physics-based, predictive modeling, management of errors and uncertainties, automation in all phases of the CFD analysis process, effective use of massively parallel, heterogeneous, and fault-tolerant High Performance Computing (HPC) architectures that will be available in the 2030 time frame, balanced and flexible utilization of HPC systems, and seamless integration with multidisciplinary analyses and optimizations.

Geometry preprocessing and mesh generation are cited by the Study as prominent impediments to applied CFD. Generation of suitable meshes about complex configurations is said to constitute a principal bottleneck and dominant cost in the CFD process. Current mesh generation software is said to be unable to consistently produce valid, high-quality meshes of the desired resolution about complex configurations on the first attempt. Coupling and linking of geometry models to both mesh generation and CFD software is said to be lacking. Geometry models themselves are said to be poorly suited for CFD. Mesh generation software is said to not scale appropriately on parallel computer architectures.

Subsequent to the Study's publication, the membership of AIAA's Meshing, Visualization, and Computational Environments (MVCE) technical committee (TC) agreed to take responsibility for stewardship of the Study's citations and recommendations that fell within its charter, including those related to geometry preprocessing and mesh generation. The MVCE TC began its Study-related work by hosting panel discussions on geometry and meshing (AIAA SciTech Forum 2015, Reference 2), visualization (AIAA Aviation Forum 2015, Reference 3), and computational environments (AIAA SciTech Forum 2017, summary not yet published).

The geometry and meshing panel discussion in 2015 confirmed all of the Study's commentary on both topics but with additional insights. While it was virtually unanimous that working with Computer-Aided Design (CAD) data was meshing's Achilles heel, it became clear that $\mathrm{CAD}$ data represents multiple challenges. One challenge is the need to work with analytic (i.e. non-uniform rational B-Splines) and/or discrete (i.e. faceted) geometry models based on users' requirements. The underlying technologies of the two geometry types have little overlap. Another challenge is the simple interoperability of geometry model files. The annual cost of Computer-Aided Engineering (CAE) data interoperability problems in just the U.S. automotive industry was at least \$1 billion in 1999 (Reference 4); the authors have no reason to believe this differs for the U.S. aerospace industry or that the situation has improved in the decades since. Yet another challenge may be derived from using a geometry model created for manufacturing purposes (referred to in Reference 5 as an "MCAD model") versus using a model created specifically for simulation (a "pre-CAD" model in Reference 5). The difference between MCAD and pre-CAD geometry models results from differences in the purposes for which they were created. The former often includes significantly more detail than the wetted surfaces needed by CFD (for example, rivets, seams, structural elements) and often is built to tolerances orders of magnitude larger than the minimum mesh spacing. And finally, the geometry modeling challenge may arise from a simple lack of understanding of what is in a geometry model and how it gets meshed, leading to unfounded expectations (Reference 5). For example, the designers of the NASA High Lift Common Research Model (HL-CRM) geometry model (Reference 6) state that "many CFD processes expect a single surface wing as their input... the decision was made to create a one-piece wing loft." While that statement may be factually true, it is equally true that many CFD processes are agnostic to geometry model topology. Therefore, attempts to tailor a geometry model for a perceived broad audience often mask challenges (complex geometry model topology in this case) that would be better unmasked and addressed. Another example of a common modeling practice that should not be classified as a best practice is the use of structured quadrilateral surface grids (i.e. mapped meshes) as geometry. These types of bilinear surfaces are often overly dense (leading to slow computations) and often exhibit slope discontinuities within their interior that violate a primary assumption (slope continuity) within most geometry modeling software.

While working with geometry models is a challenge for all mesh generation software, each particular meshing strategy and implementation faces unique challenges. For example, creation of grid topology is unique to multiblock structured grids. Unstructured and hybrid mesh generation, while based on strong theoretical background with either Delaunay or advancing front methods, still contend with the elimination of sliver cells. The panel discussion also touched on the relative merit of newer, Cartesian-based CFD solvers that seek to eliminate the meshing burden virtually entirely.

In Reference 7, members of the MVCE TC expanded further on the geometry and meshing aspects of the Study. With respect to geometry modeling, opening a dialog between geometry, mesh, and CFD experts was the first step toward building a foundation for future work that would include developing frameworks and processes, and investigating new modeling technologies (subdivision surfaces, for example). With respect to meshing, the industry as a whole needs to properly evaluate newer methods (e.g., Cartesian, high order, polyhedral, adaptive) to effectively assess their benefits relative to the current state of the art. Regardless of the type of mesh, the effects of 
the mesh's properties on solution convergence and numerical accuracy need to be quantitatively studied. Reference 7 ends with an announcement of the $1^{\text {st }}$ AIAA Geometry and Mesh Generation Workshop (GMGW-1), the subject of this paper, as a means of creating a datum against which progress toward the 2030 vision can be measured.

\section{B. High Lift Prediction and Mesh Generation}

Recognizing the close coupling of mesh generation to the numerical accuracy and convergence behavior of current generation of Reynolds-Averaged Navier-Stokes (RANS) CFD flow solvers, intensive efforts within most, if not all, of the aerodynamic prediction workshops (for example, HiLiftPW, Drag Prediction Workshop, Aeroelastic Prediction Workshop, Propulsion Aerodynamics Workshop) have focused on generating high quality meshes (of all types) using established best practices that would be appropriate and suitable for the specific CFD simulation required. Typically, a portion of the workshop is devoted to assessing (and hopefully demonstrating) that a mesh, when properly refined, provides a "grid converged" numerical result consistent with the stated order of accuracy of the flow solver used. The so-called "asymptotic behavior" of the grid systems generated are rarely achieved, however, mainly because the requirements for proper grid refinement for very complex three-dimensional geometries are often difficult to meet. Also, consistent grid refinement is not always easy to achieve for a complex configuration, and there may be conflicting requirements between best practices and producing a consistent grid family.

When discussions began within the MVCE TC on focusing concerted effort on a more rigorous assessment of geometry and mesh generation, particularly in response to the Study, MVCE TC members reached out directly to members of the HiLiftPW committee to explore potential areas of collaboration, particularly through the mechanism of technical workshops. Through thoughtful discussion, it became evident that having the leading geometry and mesh generation experts in the aerospace community provide focused attention to the particular CFD meshing issues involved in high-lift aerodynamics could provide a potentially ground-breaking insight into the relationship between mesh and flow solution.

Throughout the three High Lift Workshops held to date, meshing has presented significant challenges. First, grid generation around realistic high lift configurations has typically been challenging, particularly when including all the bracket hardware between the slat or flap elements and main wing. Questions about the originating geometry model (and its seemingly inevitable simplifications) have raised concerns. Creation of structured grids tends to be especially time consuming, even for expert mesh generators. Unstructured grids have had their own difficulties, including problems adding sufficient clustering of grid points where needed in the wakes of each wing element (for example, where the slat wake passes over and interacts with the boundary layer of the wing and flap and where under-resolving the wake can have adverse consequences in terms of the quality of the solution). Second, although the typical sizes of grids (in terms of number of mesh cells) used for the High Lift Workshops have been gradually increasing over time, there has always been the question of whether the grids were fine enough to sufficiently eliminate the question of discretization errors overwhelming other sources of uncertainty, especially near maximum lift conditions. For example, some HiLiftPW participants have run a given code and turbulence model with different grids and obtained very different results, leading to the conclusion that the grids themselves are not adequately resolved, and therefore cannot be fully trusted (see Reference 8). Finally, to date it has been unclear what specific factors constitute a mesh of sufficient quality for capturing the most important flow physics of high-lift configurations. Although each workshop has issued meshing guidelines based on analysis best practices, these have mostly relied on prior (and varied) experiences from industry engineers, and not so much on rigorous quantitative assessments or error analyses. And there is also the perception that meshes may or may not possess particular "quality" features that could improve the ability of flow solvers, using those meshes, to converge to steady state, or perhaps to produce any CFD solutions at all. These mesh features have never been quantifiable.

Therefore, a collaboration between the two groups - high-lift CFD simulation experts with geometry and meshing experts - was deemed to be a very worthwhile venture. Further, developing the collaboration to lead directly to two separate co-located workshops (namely HiLiftPW-3 and GMGW-1) was important to demonstrate effective communication between the groups, as well as provide a forum to cross-pollinate the separate groups and determine technical areas to explore in future joint efforts.

\section{Mesh Generation and High Lift Prediction Workshops}

AIAA's successful series of CFD-related workshops has a lineage that begins with the Drag Prediction Workshop, which recently completed its sixth incarnation (as examples, see References 9 and 10). Other CFD workshops have involved shock wave/boundary layer interaction, propulsion aerodynamics, aeroelasticity, highorder methods, and sonic booms. The High Lift Prediction Workshop (HiLiftPW) series, which started in 2010, 
focuses on the prediction of swept, medium-to-high-aspect ratio wings for landing/take-off (high-lift) configurations.

The MVCE TC decided to launch GMGW-1 to begin collecting quantitative data on meshing and the mesh generation process and to provide a datum against which subsequent workshops could measure progress. While the MVCE TC could have gone it alone, being able to collect data on meshes and geometry that would be used in a variety of CFD solvers (versus meshing for meshing's sake) was preferred. Fortunately, HiLiftPW-3 was a willing partner, aided perhaps by the fact that their lead organizer is also the Study's lead author. It was then decided to launch the 1st AIAA Geometry and Mesh Generation Workshop (GMGW-1) and hold it concurrently and collaboratively with HiLiftPW-3 the weekend prior to AIAA Aviation Forum 2017.

GMGW (as a planned multi-year series of workshops) has three general goals: (1) assess the current state-of-theart in geometry preprocessing and mesh generation technology and software as applied to aircraft and spacecraft systems, (2) identify and develop understanding of areas of needed improvement (performance, accuracy, applicability) in geometry processing and mesh generation technology software, and (3) provide a foundation for documenting best practices for geometry processing and mesh generation.

More specifically, GMGW-1 focused on three activities: (1) generation of meshes to be provided to HiLiftPW-3 participants for their use in a grid convergence study, (2) collection of mesh and meshing process data for evaluation, and (3) creation of a baseline for measuring future progress toward GMGW goals.

\section{The High Lift CRM Wing-Body}

GMGW-1's collaboration with HiLiftPW-3 centered on the NASA High Lift Common Research Model (HLCRM) (Reference 6) Wing-Body configuration shown in Figure 1. The HL-CRM was used as one of three test cases for HiLiftPW-3. The HL-CRM represents a typical commercial transport aircraft. Its wing-body configuration used for the two workshops included a single leading-edge slat and inboard and outboard trailing edge flaps that were not attached to the wing. Because design and manufacture of the HL-CRM wind tunnel model is ongoing (and therefore no wind tunnel data were available for comparison), the two workshops used the HL-CRM for a grid convergence study only.

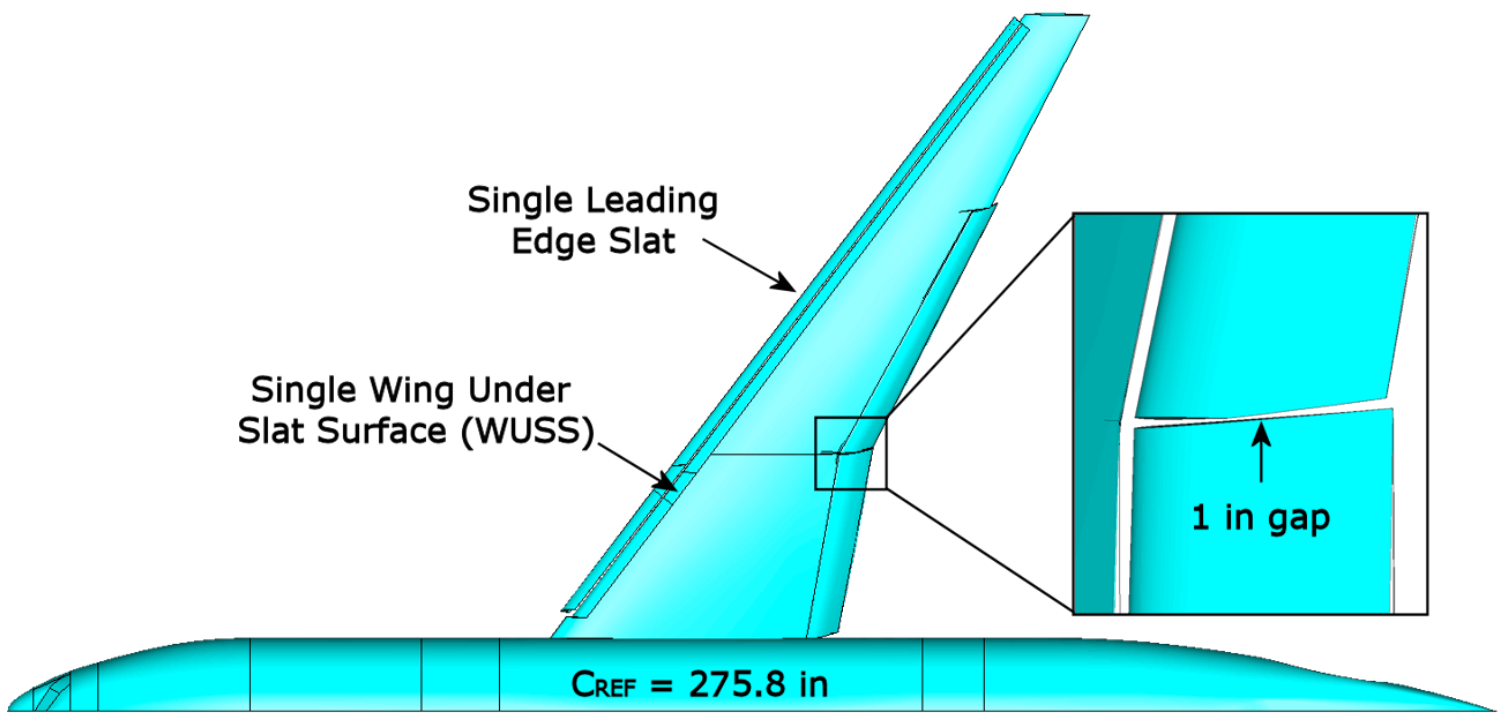

Figure 1: The High Lift CRM Wing Body Geometry Model

Workshop participants were provided with the HL-CRM geometry model in several formats including IGES (Reference 11), STEP (Reference 12), Parasolid ${ }^{\circledR}$ software (Reference 13), $\mathrm{NX}^{\mathrm{TM}}$ software (Reference 14), and $\mathrm{Creo}^{\circledR}$ software (Reference 15). Final design work of the HL-CRM was performed in Siemens NX v8.5 and therefore its native file is considered to be the definitive geometry model source. Except for the Creo file, the other formats were all exported directly from NX. The Creo file was created by importing the Parasolid file into Creo and exporting a native Creo file. The use of Creo was considered relevant because the HL-CRM wind tunnel model (to be used for HiLiftPW-4) is being designed in Creo.

There were two variants of the HL-CRM that differed in how the flaps were configured: a full gap between the inboard and outboard flaps and a partially sealed gap between the flaps (see Figure 2). The latter was a significant 
forward-looking exercise as the wind tunnel model of the HL-CRM, currently planned to be used in HiLiftPW-4, will have partially sealed flap gaps.
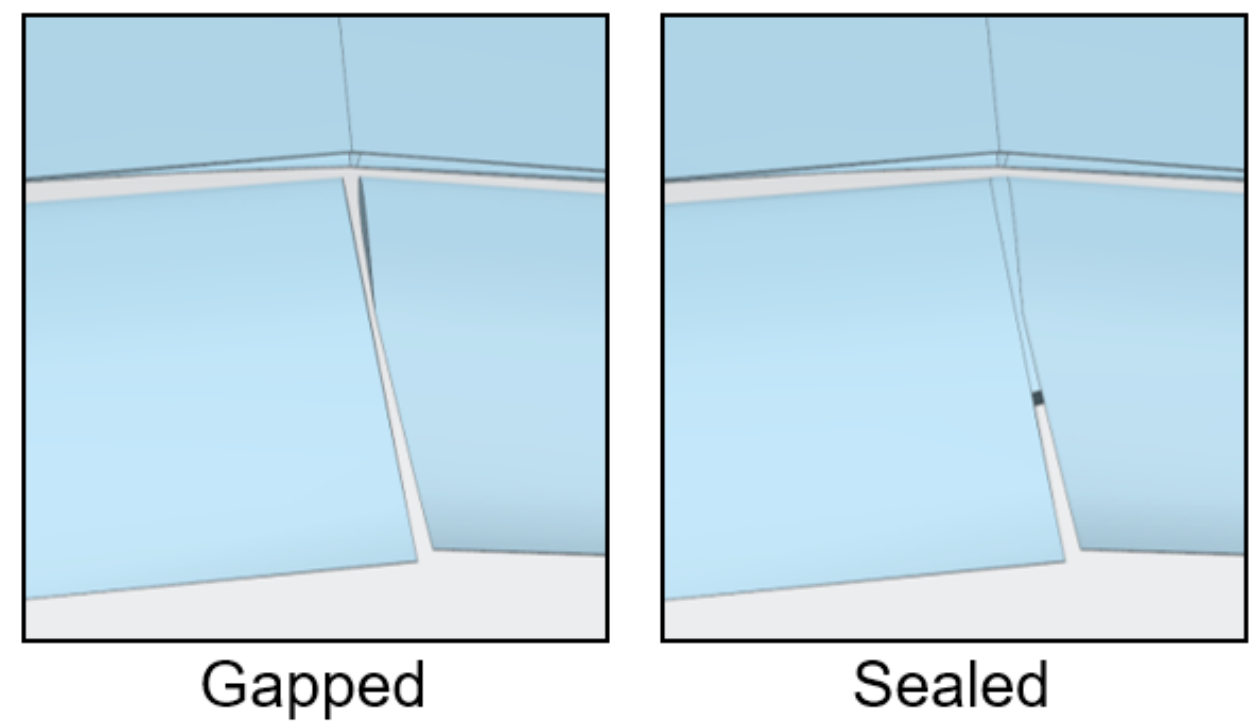

Figure 2: Comparison of the HL-CRM flap junction showing the gapped/unsealed (left) and sealed (right) variations.

\section{Generating HL-CRM Meshes for HiLiftPW-3}

The first objective of GMGW-1 was to generate families of meshes for the HL-CRM to be used for a grid convergence study by HiLiftPW-3 participants (i.e. the committee provided meshes). With a Reynolds Number of 3.26 million and a reference chord of 275.8 inches, each mesh family was to include coarse, medium, fine, and (optionally) extra fine resolution as described in Table 1.

Table 1: Specified mesh resolution criteria for the HL-CRM mesh convergence study.

\begin{tabular}{|l|c|r|r|}
\hline Resolution & Y + & Wall Normal Spacing (inches) & Mesh Points Across Trailing Edge \\
\hline Coarse & 1 & 0.00175 & 5 \\
\hline Medium & $2 / 3$ & 0.00117 & 9 \\
\hline Fine & $4 / 9$ & 0.00078 & 13 \\
\hline Extra Fine & $8 / 27$ & 0.00052 & 17 \\
\hline
\end{tabular}

Rather than prescribing a cell count, the medium resolution mesh was to be typical of those one would use in the normal course of applied CFD for a configuration of this type. Cell counts were not specified in advance and were to be a result of applying the HiLiftPW gridding guidelines, which provide desired element sizes at key locations on the vehicle such as the leading and trailing edges, wing tip, and root (for details, see Reference 16).

Within the MVCE TC, mesh generation was conducted competitively, and at one time 24 meshes or mesh families were proposed as candidates. Final selection of the meshes to be provided to HiLiftPW-3's participants was based upon successful evaluation of the following criteria which afterward evolved into the assessment criteria for GMGW-1 participants' meshes:

1. Adherence to the gridding guidelines

2. Conformance of the surface mesh to the geometry model

3. Successful evaluation of basic mesh quality metrics

4. Successful run (not to convergence) in a flow solver

Because of its status as an ISO standard designed specifically for CFD data portability, CGNS (Reference 17) was chosen as the primary format in which the committee-provided meshes were to be distributed. The structured grids were also provided in the de facto standard PLOT3D (Reference 18) format. The unstructured grids were also provided in UGRID format (Reference 19, the FUN3D CFD solver's native format) and Pointwise ${ }^{\mathbb{R}}$ format (Reference 20). Two structured and three unstructured mesh families were selected as committee-supplied meshes. 
It is important to note that the three unstructured grid families are virtually identical with respect to the grid points; the difference is whether near-wall tetrahedra were combined into layers of prisms or hexahedra.

A summary of the committee supplied meshes by designator, mesh type, flap configuration, resolution, and file format is provided in Table 2. The meshing tools used were: ANSA software (Reference 21) and Chimera Grid Tools (Reference 22) for A, GridPro software (Reference 23) for C, and Pointwise for the B series.

Table 2: The array of meshes generated by the GMGW-1 committee for use by HiLiftPW-3 participants.

\begin{tabular}{|l|l|l|l|l|l|l|l|l|l|l|}
\hline \multicolumn{3}{|c|}{ Mesh } & \multicolumn{5}{c|}{ Full Gap } & \multicolumn{5}{|c|}{ Partial Gap } \\
\hline Designator & Type & Subtype & Coarse & Med. & Fine & X-Fine & Coarse & Med. & Fine & X-Fine \\
\hline A & Structured & Overset & $\mathrm{x}$ & $\mathrm{x}$ & $\mathrm{x}$ & $\mathrm{x}$ & & $\mathrm{x}$ & & \\
\hline C & Structured & Multi-block & $\mathrm{x}$ & $\mathrm{x}$ & $\mathrm{x}$ & & $\mathrm{x}$ & $\mathrm{x}$ & $\mathrm{x}$ & \\
\hline B1 & Unstructured & Tetrahedra (Tet) & $\mathrm{x}$ & $\mathrm{x}$ & $\mathrm{x}$ & $\mathrm{x}$ & & $\mathrm{x}$ & & \\
\hline B2 & Unstructured & Tet+prism & $\mathrm{x}$ & $\mathrm{x}$ & $\mathrm{x}$ & $\mathrm{x}$ & & $\mathrm{x}$ & & \\
\hline B2 & Unstructured & Tet+hex & $\mathrm{x}$ & $\mathrm{x}$ & $\mathrm{x}$ & $\mathrm{x}$ & & $\mathrm{x}$ & & \\
\hline
\end{tabular}

Work on generating the committee meshes and the challenges faced therein was presented at AIAA SciTech Forum 2017 (References 24, 25, 26, 27). These papers and presentations are notable for their authors' candor in describing the deficiencies in their own software packages.

\section{GMGW-1 Workshop Results}

\section{A. Workshop Participation}

Fifty-one people registered for GMGW-1, a number that exceeded expectation of the MVCE TC. Of the registrants, 16 were members of the MVCE TC's Meshing Subcommittee. The registrants came to the workshop from eight countries as shown in Figure 3.

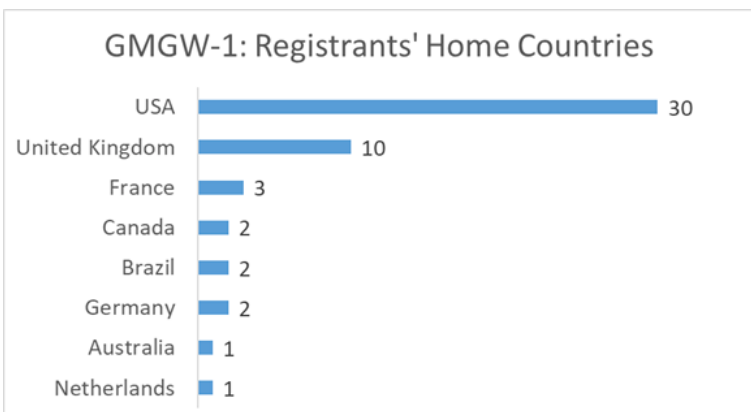

Figure 3: A breakdown of GMGW-1 registrants by their home country.

Eighteen of the registrants participated in GMGW-1, meaning that they generated meshes, submitted data, and gave presentations on their work. Three other participants withdrew prior to the workshop. Of the 18 participants, 8 were from software vendors, 6 were from industry, 2 were vendor/academia teams, and 2 were from government organizations.

Twelve participants generated their meshes using commercial software while the other participants used in-house (3) and government-supplied (2) software. Meshing tools used included (in no particular order) STAR-CCM $+{ }^{\circledR}$ software (Reference 28), ANSA, Pointwise software, Chimera Grid Tools, BOXERMesh (Reference 29), Capstone (Reference 30), GridPro, CADfix/CFD (an unpublished work in progress), Ennova (Reference 31), SLUGG (Reference 32), AGPS/AFLR3 (Reference 33), MADCAP (Reference 34), EPIC (Reference 35), SimModeler (Reference 36), and Cflow (Reference 37). In several instances, some of those meshing tools were used in combination on the same mesh.

\section{B. Committee-Supplied Meshes}

As mentioned above, a specific goal of GMGW-1 was to generate families of meshes to be provided for use by HiLiftPW-3 participants in a grid convergence study. The unstructured meshes (B1, B2, and B3 in Table 2) were the most used (in that order) followed by the overset structured grid (A). No participants used the multi-block structured grid (C), although it was made available much later than the other grids. HiLiftPW-3 participants were polled about their use of the committee meshes. 
On the positive side, no participants had to modify the committee's meshes in order to use them in their CFD solver, with the exception of one participant who had to convert the mesh's units. Solutions converged successfully on all meshes without modification to the mesh, although one user of the overset structured grids reported having to disable multigrid in their CFD solver. This is not thought to be a problem with the mesh; it is believed to be an issue in the solver's domain decomposition algorithm for parallelization.

The first problem reported was that half of the responding participants could not use the meshes in the provided format. More specifically (and perhaps alarmingly), the problems all involved the CFD software's inability to import or interpret the CGNS files as written. No conclusions have yet been reached on why importing a well-documented, ISO standard file format was so problematic. While not a specific challenge cited by the Study and not as pervasive as CAD data interoperability, CFD data interoperability should be a relatively low hurdle that the industry can solve.

Second, despite success in running and converging the CFD solution on the committee supplied meshes, approximately $70 \%$ of responding participants indicated they would have generated the meshes differently. Perhaps not surprisingly, the suggested changes were all qualitative (i.e. more or fewer points in certain regions of the surface meshes). This also proved to be true for meshes generated for the JAXA Standard Model, HiLiftPW-3's second case (with which GMGW-1 was not involved). This lack of satisfaction with mesh resolution generally stems from the difficulty of a participant flow solver to achieve mesh convergence using the committee supplied grid. While multiple levels of grid resolution were provided with each grid family, collectively the workshop participants were unable to demonstrate mesh convergence, possibly indicating that even the finest mesh levels lack sufficient resolution.

Three conclusions have been drawn from this feedback. First, if possible, the industry needs to create a quantifiable dialog on mesh clustering requirements so that they can be documented as a priori requirements, and rigorous studies need to be performed to prove that the qualitative suggestions produce quantifiable, positive results. Likely, these studies will necessarily require much higher resolution meshes than have traditionally been employed to hopefully demonstrate mesh convergence. Second, even though mesh generation experts (i.e. the MVCE TC) can generate perfectly adequate meshes that can be used to achieve convergence in flow solvers, domain expertise is required to achieve a mesh that is truly fit for a particular purpose (high lift systems, in this instance) in terms of the end user's domain expertise. These insights are specific examples of the general conclusions from Reference 7 about quantifying mesh effects on the solution, and why it appears that meshing software fails to generate a "fit for purpose" mesh on the first attempt. Thirdly, mesh adaptation needs to be more fully implemented and explored as a way to eliminate the need for these types of discussions about a priori mesh requirements as noted in Reference 38.

\section{Participant's HL-CRM Meshes}

The second objective of GMGW-1 was to have workshop participants mesh the HL-CRM and present the resulting meshes and quantified description of their meshing process at the workshop. Eighteen participants were up to this challenge, including one participant who meshed the older Common Research Model (CRM) configuration as a test of their in-work meshing technique. Three meshes generated by HiLiftPW-3 participants were also submitted for evaluation at GMGW-1. This section includes only highlights of the mesh and process assessments performed for GMGW-1; full, detailed results are included in the referenced papers. In general, the assessments include committee and participant meshes.

\section{Results - Geometry Model}

While the participants' experiences with the HL-CRM geometry model were roughly equivalent, they were not equal. All participants reported geometry model problems that required remediation prior to meshing. Yet the specific problems varied somewhat from one meshing software to the next. This is not unexpected as the various software packages use different geometry file importers and kernels for geometry evaluation. The two flaws most widely encountered were overly dense and non-uniform surface parametrizations and undulations in surface curvature. The first is illustrated in Figure 4 for the front portion of the wing-body fairing. The source of this problem is thought to be the particular choice of surface modeling technique employed to create this surface in NX. Overly dense parametrizations also caused a problem with geometry model format conversion for a participant who requested a native CATIA file. To create this file, an MVCE TC member attempted to import the STEP file into CATIA. Unfortunately, CATIA failed to import the file due to the density of the parametrization. A third-party software package was used to reparametrize the surface and export a new STEP file. That file could be imported into CATIA from which a native CATIA file was generated. However, the effect of the reparametrization on the surface's shape was not quantified. 


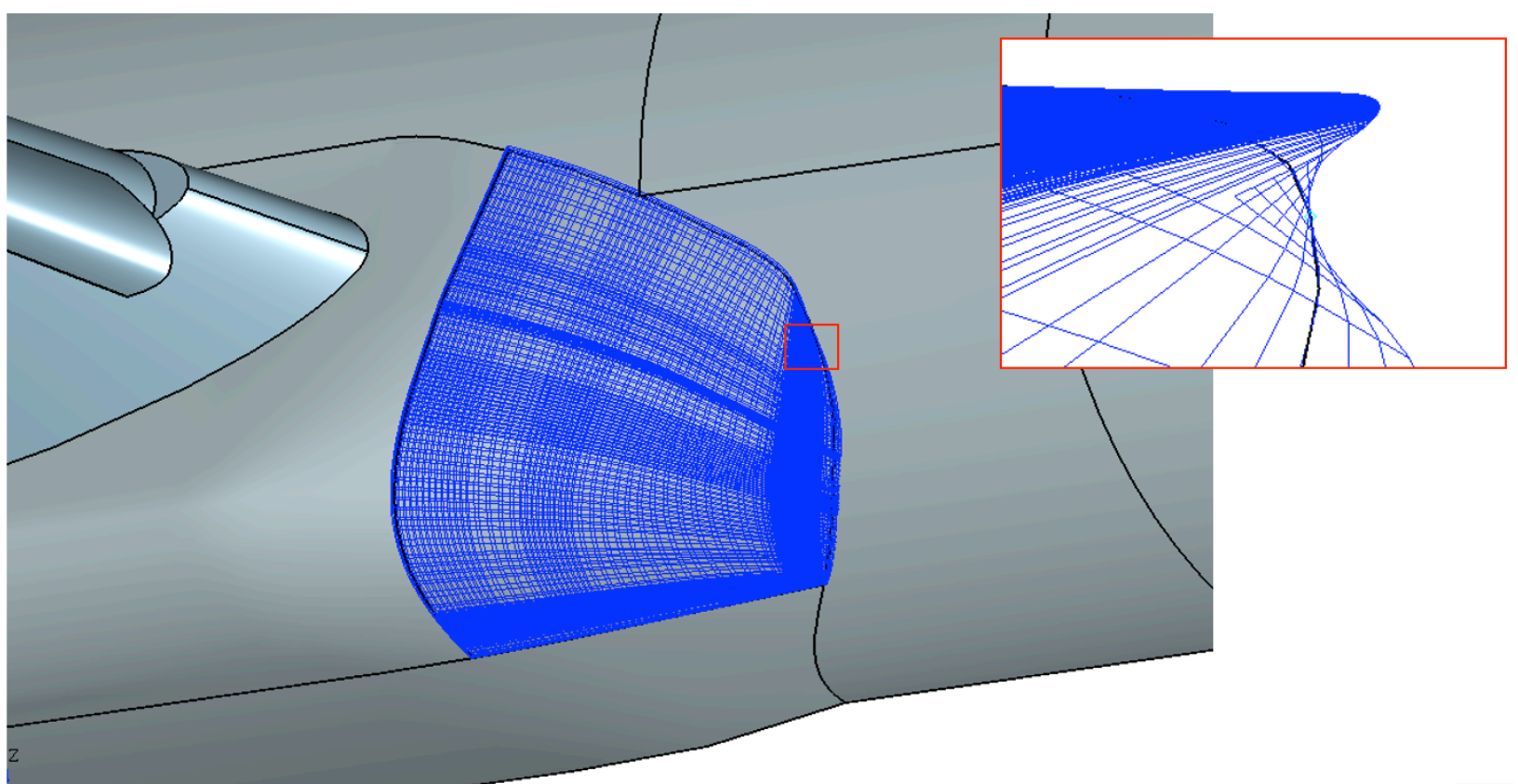

Figure 4: The forward portion of the HL-CRM's wing-body fairing used an overly dense and non-uniform parametrization as shown here. This same surface also exhibited a fold near its leading edge (see inset). Image from Reference 39.

The geometry problem regarding undulating curvature is illustrated by the fringe plot in Figure 5. The source of this problem is thought to be use of a complex surface type when a cylinder would have been equally accurate yet simpler. In particular, this surface is thought to have originated as a structured quadrilateral surface mesh output from an aerodynamic design system.

From the interoperability aspect of geometry modeling, the majority of participants used the IGES file with a few others using the STEP or Parasolid formats. Use of IGES runs contrary to current thinking on geometry model interoperability as IGES supports fewer entity types and does not handle topology information as cleanly as other, more modern storage formats. The NX file, being the native file of the originating CAD software, should have been preferred as it would require fewer error-inducing translations in the process of going from CAD software to meshing software. For example, NX-based interoperability would require as few as one translation, from NX file to meshing kernel (note the assumption that NX software to NX file is not a translation). Use of any intermediate file would require at least two translations: NX to intermediate file and file to meshing software. The situation becomes even more complex if third party software sits between the CAD software and meshing software for translation (NX to file, to interoperability kernel, to meshing kernel.) Even ignoring the benefits of using a native file, the fact that the much pilloried IGES format was favored over the more modern STEP standard is counterintuitive and requires further exploration to determine a root cause for this preference. 


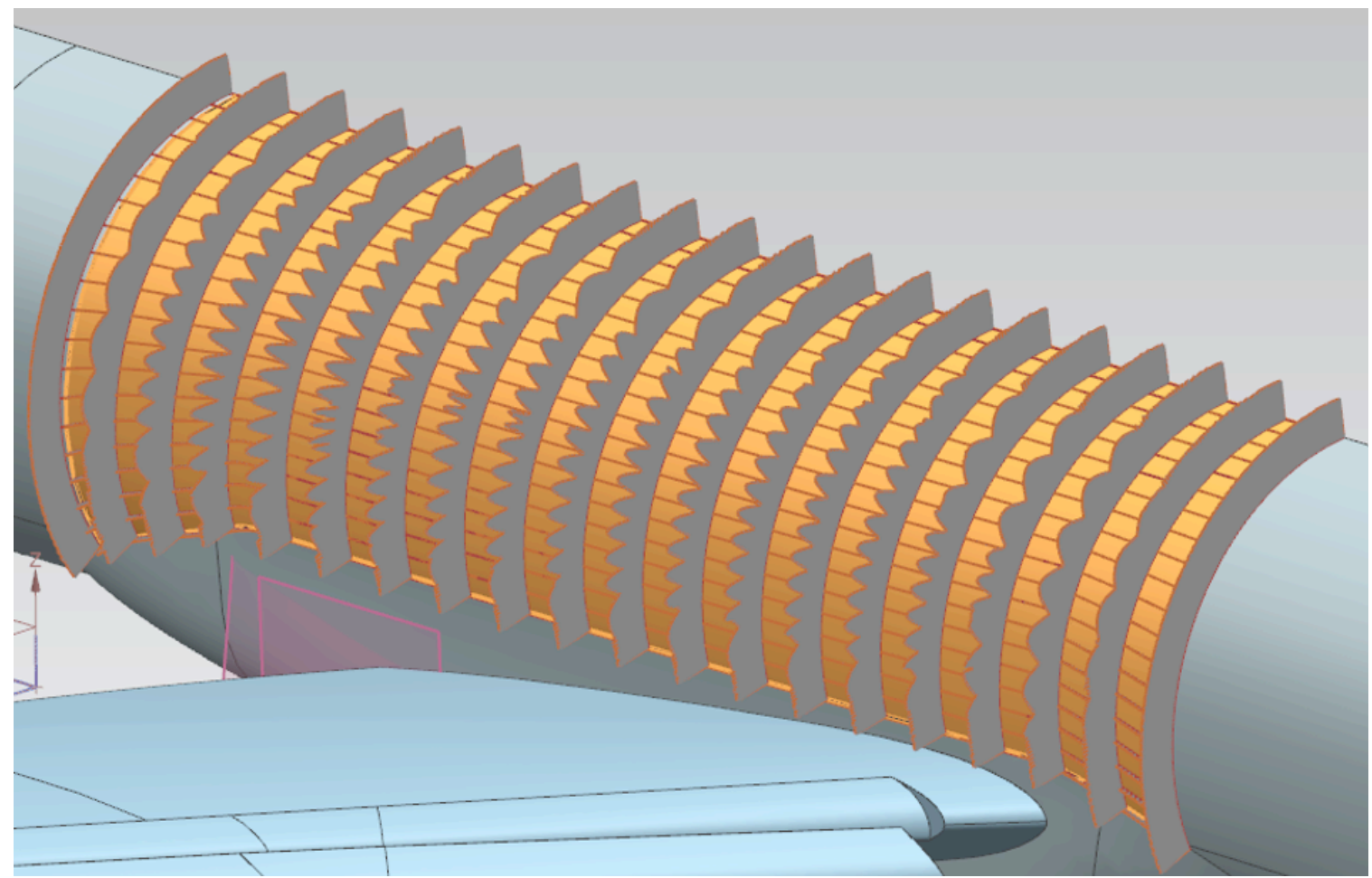

Figure 5: This fringe plot shows undulations in surface curvature on the upper portion of the HL-CRM center fuselage. Image from Reference 39.

\section{Results - Structured Grids}

Assessing the structured grids (Reference 40) involved answering questions about validity and quality. Assessing validity involved determining whether the flowfield volume was filled without degeneracies, whether surface mesh points were actually on the geometry model (where appropriate), and whether all components of the geometry model were resolved by mesh. Assessing mesh quality involved determining whether the gridding guideline's clustering constraints were followed, and whether two representative cell metrics, stretching ratio and skew, were within an acceptable range.

Mesh quality was easier to assess than validity. For the purposes of GMGW-1, stretching ratio at a point was defined as the ratio of adjacent mesh edges aligned in the same computational coordinate direction. Acceptability was defined as a value in excess of 0.8 . Skew was defined as the ratio of the cell's diagonals with acceptability again defined as a value in excess of 0.8 . Generalizations about structured grids cannot be made due to the low number (3) of grids evaluated. However, all three failed the skew test with roughly $40 \%$ of cells having skew values less than 0.8 . Without an assessment of that metric's influence on CFD solver performance, it remains to be determined whether that metric is relevant to structured grid solver performance. The grids exhibited better stretching ratio values relative to the acceptable target with the committee's overset grid meeting the criterion except for approximately $1 \%$ of the points for the medium-resolution, partially-sealed gap case. The committee's multi-block structured grid exhibited more non-smoothness with approximately $5 \%$ of points exceeding the criterion. This might be explained by the point matching topology requirements of a multi-block versus an overset grid which cause the grid lines to take more circuitous paths.

More challenging was the assessment of validity, and specifically whether surface grid points conformed to the geometry model (i.e. were located sufficiently close to it, ideally within computational round off error). In order to conduct this assessment, new software was written that imported the HL-CRM STEP file and grid, projected each surface grid point onto the geometry model, measured the closest-point difference between the grid point and geometry model, and identified the surface patch within the geometry model to which the grid point was projected. Two of the three structured grids were generated using the same overset gridding software and that software used a triangular faceted version of the HL-CRM geometry model with the assumption that the faceting was sufficiently dense to be a close approximation to the actually parametric NURBS model. That assumption proved to be false as distances from grid point to geometry were found to be as high as 0.5 . Later analysis of the flow solution revealed oscillations in certain regions that did not appear when the mesh was regenerated using the NURBS model. 
Regardless, during the selection process for the committee supplied meshes this assessment identified nonconforming meshes (in all cases resulting from user oversights) that were corrected before final meshes were generated. Most frustrating about this entire assessment process is the fact that the meshing software knows precisely where each surface grid point is on the geometry model yet that data is rarely transferred downstream to the CFD solver and other applications. Downstream users must resort to recreating that mesh-geometry associativity in a computationally intensive process, assuming they even have a geometry kernel that can perform it.

\section{Results - Unstructured Meshes}

Similar to the assessment of the structured grids, unstructured meshes were evaluated for validity (topologically sound without negative volumes, conformance to gridding guidelines, geometry model adherence) and quality (volume ratio). A representative assessment of guideline conformance is shown in Figure 6 where mesh spacing on the wing surface (i.e. skin spacing) is shown for several meshes. The gridding guidelines specified edge lengths at the leading and trailing edges of 0.275 inches (approximately $1 \%$ of chord) and 1.0 inches at the root and tip. All meshes with one exception met these criteria. The same can be said of the mesh quality assessment of volume ratio with an additional observation; the largest volume ratios nearly always occurred where cell type changed from the near-wall, semi-structured cells (prisms and hexahedra) to the farfield cells (pyramids and tetrahedra).

With respect to the assessment of mesh cell quality metrics for both the unstructured and structured grids, the experience for GMGW-1 identified where additional research is needed. First, there is no clear correlation between a specific metric (stretching ratio, for example) and the flow solver's convergence or accuracy other than general best practices. A more rigorous understanding of this relationship could significantly decrease or change the level of effort required to generate a suitable mesh on the first attempt. Second, in order to more effectively communicate and share metric information, a consistent vocabulary (name, formulation, method of computation, acceptable value range, method of presentation or tabulation) needs to be developed.
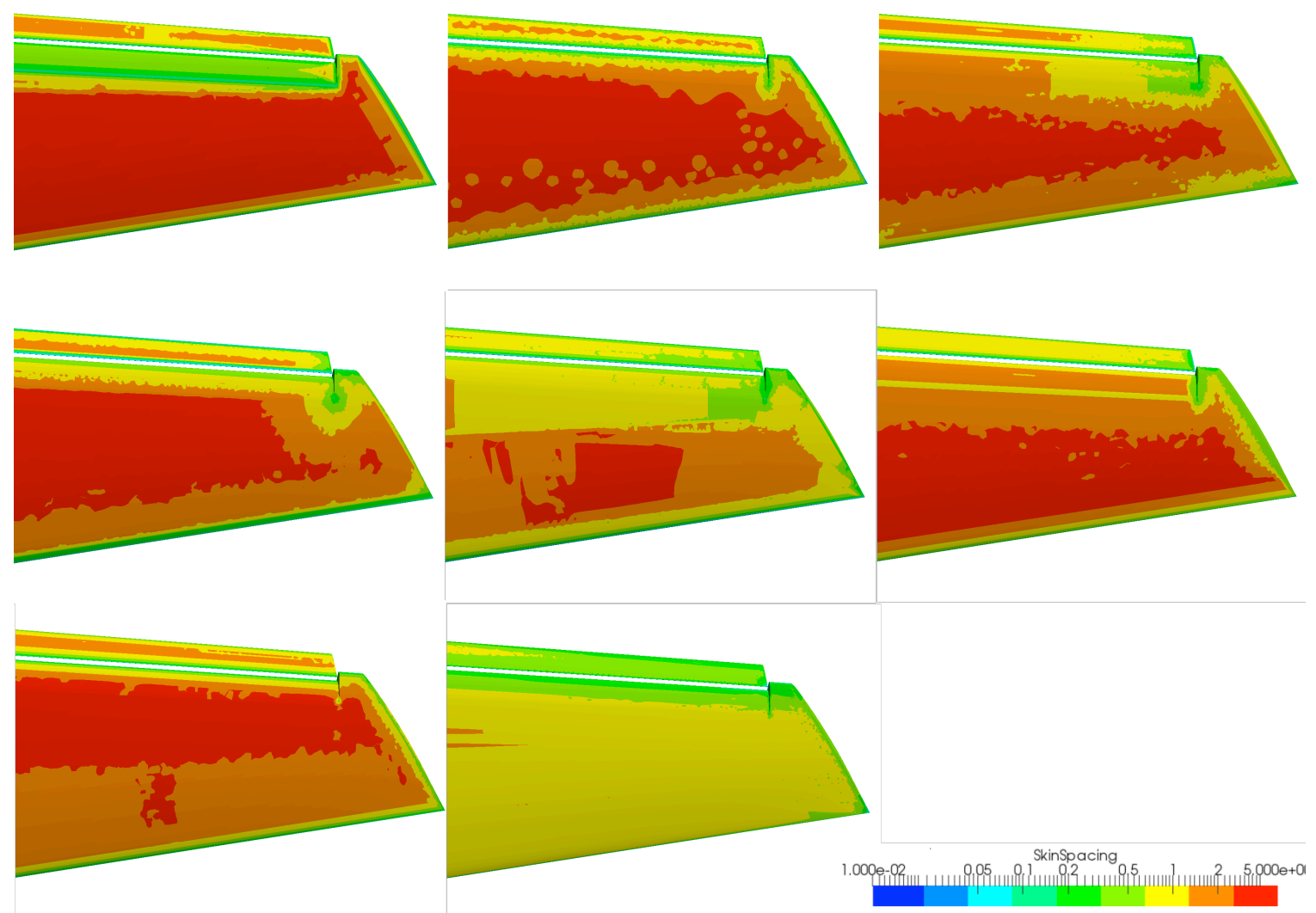

Figure 6: Comparison of mesh spacing on the wing upper surface near the tip for several medium-resolution unstructured meshes. Units in inches. Image from Reference 41.

The assessment of the unstructured mesh's geometry model adherence was conducted in a similar manner to the same assessment of the structured grids with one major difference: the new tool developed for this purpose used a different geometry kernel than was used for the structured grids. The impact of the latter is significant. The chart in 
Figure 7 illustrates the computed range of distances from surface grid points to geometry model. Note that all the distances for the three meshes by Woeber (i.e. Pointwise) are all below $10^{-12}$. However, the kernel used in the tool written for this assessment used Pointwise's geometry kernel. Therefore, it makes sense that the Pointwise mesh would appear to adhere exactly to the geometry model since the computations are identical to those performed during the meshes' generation. The inference to be made is that the geometry kernels used by the other mesh generators "see" the geometry differently and while internally they may be exactly accurate, when evaluated using a different kernel they appear to be less so.

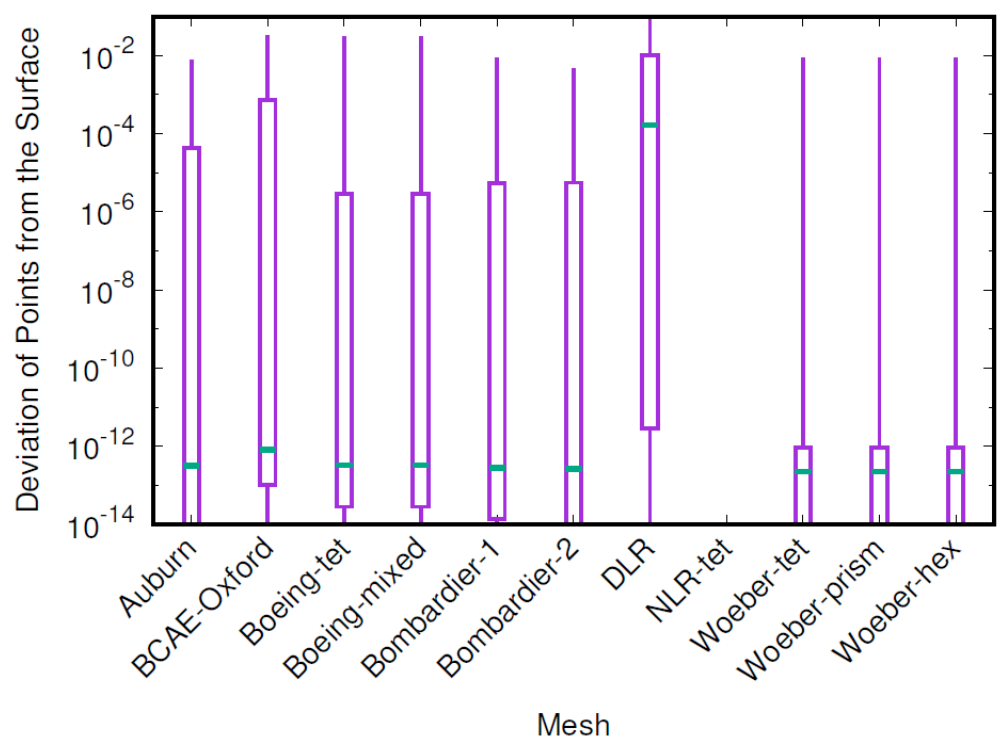

Figure 7: Evaluation of mesh adherence to the geometry model showing distance from grid points to geometry model for medium resolution unstructured meshes (listed by participant name). Image from Reference 41.

\section{Results-Meshing Process}

Each GMGW-1 participant was required to complete and submit a participant questionnaire (PQ) detailing their meshing process including the wall clock time required to complete various portions of the process. The PQ included specific questions about geometry preprocessing, surface meshing, volume meshing, mesh adaptation (if used), adherence to gridding guidelines, mesh quality metrics used, other best practices used, user expertise required for each step of the process, wall clock time required for each step of the process, specific tools used to generate the mesh families, any required rework, and file exchange issues.

All participants expended effort on preprocessing the geometry model after import to address a variety of issues (see discussion above). While most participants reported spending less than one hour on this task, an intermediate level of geometry expertise was required. 


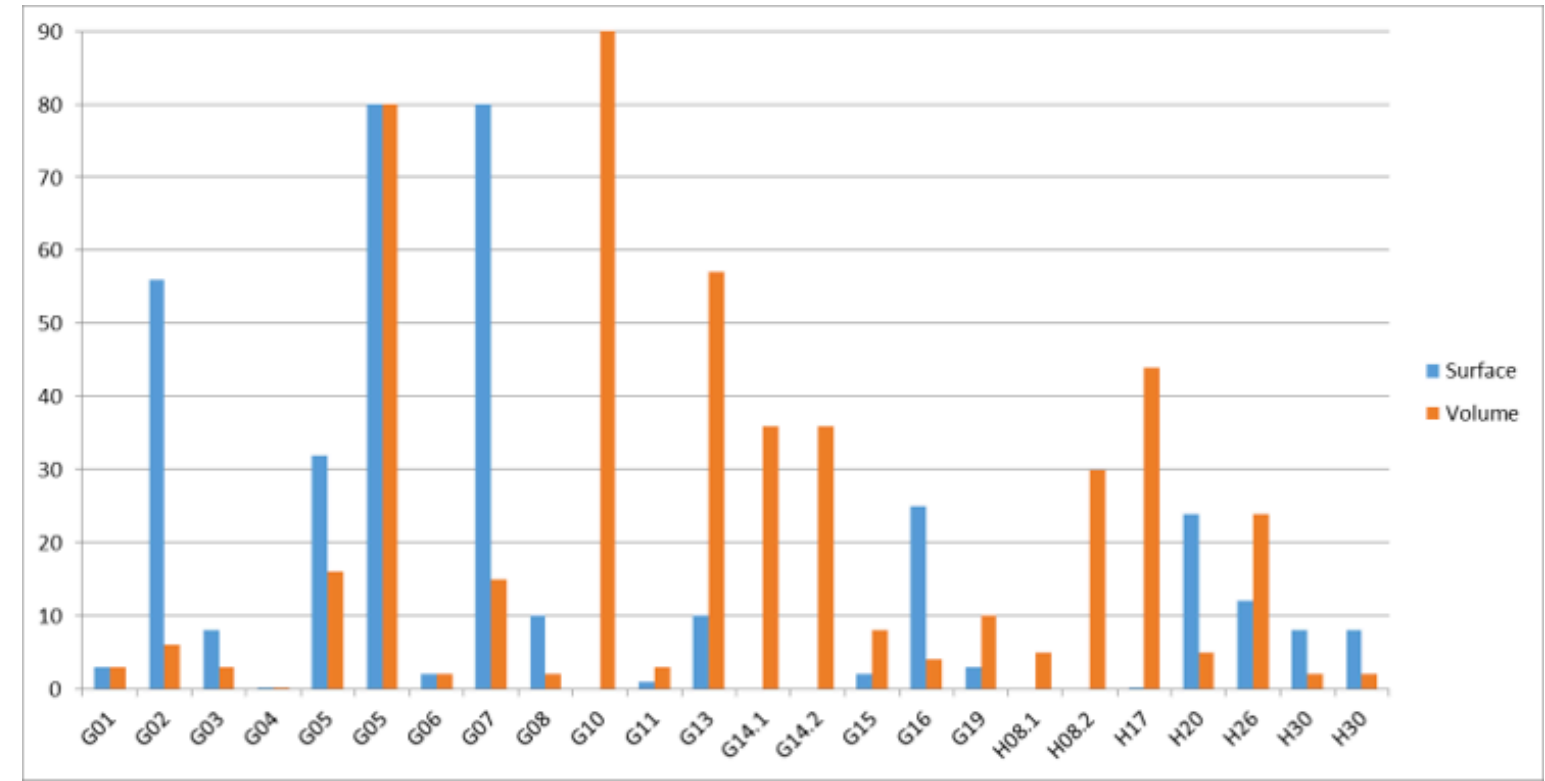

Figure 8: Meshing time (surface meshing in blue, volume meshing in orange) as reported by GMGW-1 participants. Vertical axis is hours. Image from Reference 42.

Surface meshing time ran the gamut from minutes to more than two weeks (Figure 8). At the high end of that range are the structured grid generators. At the low end of the time range are techniques where the surface mesh is generated as a by-product of the volume mesh. The two extremes of the time range are typified by two presentations in particular, one by a software vendor and one by that vendor's customer. The vendor took time to explore all the intricacies of the process interactively, driving up meshing time. On the other hand, the customer had automated their use of the vendor's code and delivered a surface mesh in mere minutes. Volume meshing time showed a similar spread from minutes to days. The expertise required for the two meshing tasks skewed higher than that required for geometry preprocessing, with more expert-level knowledge required.

Of note relative to the Study's critique of meshing, no participant generated a mesh in their first and only attempt. After the first attempt, the mesh was regenerated to better account for mesh size progression and (most importantly) adding mesh resolution for the wakes (i.e. something other than the geometry model).

\section{GMGW-1 Summary and Future Plans}

The 1st Geometry and Mesh Generation Workshop met its goals and therefore can be classified as a success. Fifty-one participants were fully engaged in two days of frank, detailed presentation and discussion of the current state of geometry preprocessing and mesh generation for CFD. Meshing experts presented in detail where their meshing software, tools, and methods succeeded and, perhaps more significantly, failed for the NASA High Lift Common Research Model wing-body configuration. Mesh families were generated for the HL-CRM and provided to High Lift Prediction Workshop participants who used them in a grid convergence study. Analyses of geometry modeling practices, structured and unstructured mesh quality, and the mesh generation process were conducted. These analysis results will form the datum against which future GMGW events can measure progress.

As the first workshop of its type, GMGW-1 mostly confirmed aspects of the geometry modeling and mesh generation processes that were already known. The workshop experience did, however, reveal specific aspects of those processes that should be further explored and answers to the following questions sought:

- How can we eliminate geometry modeling practices that are detrimental to meshing?

- Why does IGES remain the preferred geometry model exchange format and is that a bad thing?

- Can mesh-geometry associativity information be shared by the meshing software with downstream applications?

- Through coordination with the CGNS steering committee, can we eliminate mesh file interoperability problems by documenting best practices?

- What mesh quality metrics are significant and therefore worth computing and evaluating?

- Should the current gridding guidelines for CFD workshops be updated and if so, how? 
- When it comes to adherence of the surface mesh to the geometry, what are the implications of the software used for exchanging and evaluating geometry models?

It is important to note that at least one participant employed mesh adaptation for the HL-CRM. While that particular mesh was not explored in this paper, continued development of adaptation is significant relative to the NASA CFD Vision 2030 Study's goals. Mesh-geometry associativity information (see third bullet above) is an enabling technology for surface mesh adaptation. An Unstructured Grid Adaptation Working Group has been formed (independent of the MVCE TC) and published their first benchmark (Reference 43). Work of this type should be monitored by and perhaps more fully incorporated into future GMGW workshops.

Planning is already underway for GMGW-2. Lessons learned from GMGW-1 will be applied to the execution of subsequent workshops. While the details of GMGW-2 have not yet been fully defined, its implementation should improve upon the following issues.

- Evaluate procedurally a geometry model to identify potential problems in advance.

- Develop a consistent and rigorous methodology for evaluating and reporting any surface mesh's conformance to a geometry model.

- Document a dictionary of mesh metrics.

- Develop a consistent and rigorous methodology for evaluating and reporting any mesh's quality metrics.

- Encourage participation in future workshops from non-traditional meshing technologies (what is referred to as meshing's "Kodak moment" in Reference 2).

- Work closely with CFD workshops to refine their gridding guidelines and more fully quantify clustering requirements.

- Integrate the results of mesh adaptation in the workshop's data collection.

- Focus more closely on measuring the many aspects of the overall process.

In addition to planning for GMGW-2, other actions have already been taken in the wake of GMGW-1. An updated version of the geometry model for the HL-CRM configuration was created to eliminate the modeling problems cited above. This updated model configuration is available for future uses, and will be used for 5 th International Workshop on High Order CFD Methods (Reference 44) in which it is one of two meshing challenge cases.

To begin addressing the technical impediments to geometry preprocessing and mesh generation, the MVCE TC has formed working groups on these two subjects, respectively, with the goal of creating best practices documents that can form the basis for future workshops.

Beyond GMGW-2, very preliminary discussions have been held regarding GMGW-3 with hopes to collaborate with HiLiftPW-4, which is expected to compare CFD simulations with semi-span HL-CRM configuration wind tunnel model data being collected at NASA, and potentially full-span configuration data collected at other facilities. Another joint, collaborative workshop experience like this will allow us collectively to build on the efforts invested in GMGW-1 ${ }^{\mathrm{a}}$ and HiLiftPW-3.

\section{References}

1. Slotnick, J., Khodadoust, A., Alonso, J., Darmofal, D., Gropp, W., Lurie, E., and Mavriplis, D., "CFD Vision 2030 Study: A Path to Revolutionary Computational Aerosciences," NASA/CR-2014-218178, March 2014.

2. Chawner, J.R., Dannenhoffer, J.F., Dey, S., Thornburg, H., Jones, W.T., Slotnick, J., and Taylor, N.J., "The Path to and State of Geometry and Meshing in 2030: Panel Summary," AIAA 2015-3409, January 2015.

3. Duque, E., Imlay, S., Ahern, S., Chen, G., and Kao, D., "NASA CFD Vision 2030 Visualization and Knowledge Extraction: Panel Summary from AIAA Aviation 2015 Conference," AIAA-2016-1927, January 2016.

4. Brunnermeier, S.B., and Martin, S.A., "Interoperability Cost Analysis of the U.S. Automotive Supply Chain," Research Triangle Institute Project Report No. 7007-03, March 1999.

5. Taylor, N., "Industrial Perspectives on Geometry-Handling for Aerodynamics," AIAA 2015-3408, June 2015.

6. Lacy, D., and Sclafani, A., "Development of the High Lift Common Research Model (HL-CRM): A Representative High Lift Configuration for Transonic Transports," AIAA 2016-0308, January 2016.

7. Chawner, J.R., Dannenhoffer, J.D., and Taylor, N., "Geometry, Mesh Generation, and the CFD 2030 Vision," AIAA 20163485, June 2016.

\footnotetext{
${ }^{a}$ Presentations from GMGW-1 and announcements regarding future GMGW events can be found on the workshop's
} website, www.gmgworkshop.com. 
8. Rumsey, C. L., Slotnick, J. P., and Sclafani, A. J., "Overview and Summary of the Third AIAA High Lift Prediction Workshop," AIAA SciTech Forum 2018 (submitted for publication).

9. Tinoco, E.N., Brodersen, O.P., Keye, S., Laflin, K.R., Feltrop, E., Vassberg, J.C., Mani, M., Rider, B., Wahls, R.A., Morrison, J.H., Hue, D., Griepy, M., Roy, C.J., Mavriplis, D.J., and Murayama, M., "Summary of Data from the Sixth AIAA CFD Drag Prediction Workshop: CRM Cases 2 to 5," AIAA 2017-1208, January 2017.

10. Roy, C.J. "Summary of Data from the Sixth AIAA CFD Drag Prediction Workshop: Case 1 Code Verification," AIAA 20171206, January 2017.

11. Reed, K., Kelly, J.C., Harrod, D., Conroy, W., “The Initial Graphics Exchange Specification (IGES) Version 5.1,” U.S. National Institute of Standards and Technology, NIST 4412, September 1991.

12. "STEP Application Handbook ISO 10303 Version 3," SCRA, June 2006.

13. Parasolid, software package, Version 23.1, Siemens PLM Software, Plano, Texas, 2017. Parasolid is a registered trademark of Siemens Product Lifecycle Management Software Inc. or its subsidiaries in the United States and in other countries.

14. NX, software package, Version 11, Siemens PLM Software, Plano, Texas, 2016. NX is a trademark of Siemens Product Lifecycle Management Software Inc. or its subsidiaries in the United States and in other countries.

15. Creo, software package, Version 4, Needham, Massachusetts, 2017. PTC and Creo are trademarks or registered trademarks of PTC Inc. or its subsidiaries in the U.S. and in other countries.

16. "3rd AIAA CFD High Lift Prediction Workshop Gridding Guidelines," URL: https:/hiliftpw.larc.nasa.gov/Workshop3/GriddingGuidelines-HiLiftPW3-v10.pdf [cited 06 October 2017].

17. Rumsey C.L., Wedan B., Hauser T., and Poinot M., "Recent Updates to the CFD General Notation System (CGNS)", AIAA Paper 2012-1264, January 2012.

18. "Plot3d File Format for Grid and Solution Files," URL: https://www.grc.nasa.gov/www/wind/valid/plot3d.html [cited 06 November 2017].

19. "FUN3D Fully Unstructured Navier-Stokes,” URL: https://fun3d.larc.nasa.gov/, October 2017.

20. Pointwise, software package, Version 18, Pointwise, Inc., Fort Worth, Texas, 2017. Pointwise is a registered trademark of Pointwise, Inc. in the USA and the EU.

21. “ANSA, META for Multidisciplinary CAE Pre- and Post-Processing,” URL: http://www.ansa-usa.com/wpcontent/uploads/2017/04/ansa_meta_brochure.pdf [cited 06 November 2017].

22. Chan, W.M., Rogers, S.E., Pandya, S.A., Kao, D.L., Buning, P.G., Meakin, R.L., Boger, D.A., Nash, S.M., "Chimera Grid Tools User's Manual," Version 2.1, March 2010, URL: https://www.nas.nasa.gov/publications/software/docs/chimera/index.html [cited 06 November 2017].

23. GridPro, software package, Version 6.6, Program Development Company, White Plains, New York, 2017. GridPro is a trademark or registered trademark of the Program Development Company, LLC or its subsidiaries located in the United States or other countries.

24. Li, P., Gallegos, J., and Michal, T., "Generation of Mixed Element Unstructured Grids for the $3^{\text {rd }}$ High Lift Prediction Workshop," AIAA 2017-0361, January 2017.

25. Chan, W.M., "Best Practices on Overset Structured Mesh Generation for the High-Lift CRM Geometry," AIAA 2017-0362, January 2017.

26. Woeber, C.D., Gantt, E.J.S., and Wyman, N.J., "Mesh Generation for the NASA High Lift Common Research Model (HLCRM)," AIAA 2017-0363, January 2017.

27. Dey, S., Aubry, R., Karamete, K., Mestreau, E.L., Dean, J.L., and Richardson, M., "Mesh Generation for High-Lift Aircraft Geometry Configurations," AIAA 2017-3064, June 2017.

28. STAR-CCM+, software package, Version 12, Plano, Texas, 2017. STAR-CCM+ is a trademark of Siemens Product Lifecycle Management Software Inc. or its subsidiaries in the United States and in other countries.

29. BOXERMesh, software package, Version 3.7, Cambridge, UK, 2017.

30. Capstone, software package, U.S. DoD High Performance Computing and Modernization Program, 2017.

31. Ennova, software package, Ennova Technologies Inc., Berkeley, California, 2017.

32. SLUGG, System for Low-speed Unstructured Grid Generation, software package, Boeing Research \& Technology, 2017.

33. AGPS/AFLR3, software packages, Boeing Research \& Technology, 2017.

34. MADCAP, software package, Boeing Research \& Technology, 2017.

35. EPIC, software package, Boeing Research \& Technology, 2017.

36. SimModeler, software package, Simmetrix, Inc., Clifton Park, New York, 2017.

37. Cflow, software package, Kawasaki Heavy Industries Ltd., Japan, 2017.

38. Park, M.A., Krakos, J.A., Michal, T., Loseille, A., and Alonso, J.J., "Unstructured Grid Adaptation: Status, Potential Impacts, and Recommended Investments Toward CFD Vision 2030," AIAA 2016-3323, June 2016.

39. Taylor, N., Jones, W., and Gammon, M., "Preparation and Analysis of Geometry Models Used in the AIAA Geometry and Mesh Generation I and High Lift Prediction III Workshops," AIAA SciTech Forum 2018 (submitted for publication).

40. Dannenhoffer, J.F., "Assessment of Structured Grids," AIAA SciTech Forum 2018 (submitted for publication).

41. Ollivier-Gooch, C., "Analysis of Unstructured Meshes from GMGW-1/HiLiftPW-3," AIAA SciTech Forum 2018 (submitted for publication).

42. Taylor, N. "Analysis of Participant Questionnaires Submitted to the $1^{\text {st }}$ AIAA Geometry \& Mesh Generation Workshop," AIAA SciTech Forum 2018 (submitted for publication). 
43. Ibanez, D., Barral, N., Krakos, J., Loseille, A., Michal, T., and Park, M., "First Benchmark of the Unstructured Grid Adaptation Working Group," $26^{\text {th }}$ International Meshing Roundtable, Procedia Engineering, 2017.

44. HiOCFD5: $5^{\text {th }}$ International Workshop on High-Order CFD Methods, URL: https://how5.cenaero.be/ (cited October 2017). 